\title{
Karpaltunnelsyndrom bei intensiver Smartphone-Nutzung
}

\author{
Korrespondenzadresse \\ Prof. Dr. Dr. Manfred Spitzer \\ Universität Ulm \\ Abteilung für Psychiatrie \\ Leimgrubenweg 12-14 \\ $87054 \mathrm{Ulm}$
}

\author{
Bibliografie \\ DOI https://doi.org/10.1055/a-0949-1354 \\ Nervenheilkunde 2019; 38: 712-713 \\ (c) Georg Thieme Verlag KG Stuttgart · New York \\ ISSN 0722-1541
}

Es gibt mittlerweile zwar eine Reihe von Übersichten zu muskuloskelettalen Symptomen bei übermäßiger Nutzung digitaler Endgeräte [3, 7, 13], vom Handy-Daumen über den Smartphone-Nacken und den Computer-Arbeitsplatz-Rücken. Das Karpaltunnelsyndrom gehörte bislang nicht zu dieser Liste [10]. Dies hat sich geändert, wovon die folgende kleine Übersicht handelt.

Mehrere in den letzten Jahren international erschienenen Studien zeigen, dass es bei intensiver Nutzung von Smartphones zu Schmerzen und Taubheitsgefühlen im Bereich der Finger und der Hand kommen kann. Ursache ist das schon lange bekannte Karpaltunnelsyndrom: Vom Unterarm zur Hand verläuft auf der Handflächenseite des Handgelenks eine tunnelartige, aus Bindegewebe bestehende Röhre, der Karpaltunnel, durch den neben 9 Sehnen zum Beugen der Finger auch der Nervus medianus verlaufen. Ist der Tunnel ohnehin schon eng und kommen noch vielfache monotone oder ungewöhnliche Handbewegungen dazu, kann es durch eine Reizung des Nerven zu Schmerzen im Bereich der Hand und zu einem Taubheitsgefühl und Kribbeln im Zeigefinger und Mittelfinger der betroffenen Hand kommen. Dies tritt besonders nachts auf, sodass viele Patienten beim Aufwachen annehmen, dass sie ungünstig gelegen haben und die Hand „eingeschlafen“ ist. Später können eine Muskelschwäche am Daumenballen und chronische bis in den Arm ziehende heftige Schmerzen hinzukommen (Brachialgia nocturna paraesthetica).

Das Karpaltunnelsyndrom - weil das Wort so lang ist spricht man meist vom „KTS“ (oder verwendet die englische Abkürzung „CTS“) - kommt bei Frauen etwa 3-mal häufiger vor als bei Männern, häufig auch in der Schwangerschaft. Dies liegt an einem kleineren und zudem flacheren Tunnel, wodurch der Nerv eher beengt wird [8]. Bei Kindern (mit noch kleinerem Tunnel) kommt das KTS allerdings praktisch nicht vor. Bestimmte Berufe dagegen gehen mit einem erhöhten Risiko, ein KTS zu entwickeln, einher: Metzger, die viel mit Messern hantieren, Reinigungskräfte, die oft Wäsche auswringen, oder Leute, die sehr viel Zeit mit Stricken oder Klavierspielen verbringen, leiden mit höherer Wahrscheinlichkeit an einem KTS, das u. a. deswegen bei Metzgern oder Musikern als Berufskrankheit anerkannt werden kann.

Man erkennt das KTS klinisch, denn oft ist dabei der Karpaltunnel auf Druck und leichtes Beklopfen empfindlich, und der Patient verspürt Schmerzen oder das genannte Kribbeln in Zeige- und Mittelfinger. Dieses Phänomen ist als Hoffmann-Tinel-Zeichen bekannt. Ein weiterer klinischer Test besteht in der Überstreckung oder starken Beugung des Handgelenks, wobei es ebenfalls zu Missempfindungen im sensiblen Versorgungsgebiet des Nervus medianus kommt (Phalen-Zeichen). Bei entsprechendem Verdacht wird die motorische Überleitungszeit des Nervus medianus zwischen dem Stimulationsort am Handgelenk und der von diesem Nerv innervierten Daumenballenmuskulatur gemessen. Falls diese distale motorische Latenz bei über 4,2 Millisekunden liegt, wird von einer Schädigung des Nervus medianus ausgegangen. Auch Messungen der sensiblen Nervenleitgeschwindigkeit, entweder im Seitenvergleich oder im Vergleich mit dem Nervus ulnaris können die Diagnose erhärten. Die Therapie reicht von Ruhigstellung, etwa durch eine Schiene am Handgelenk (besonders nachts) über Injektionen bis zur operativen Spaltung der Röhre (Retinaculum flexorum).

Dies alles ist lange bekannt und würde hier nicht so ausführlich geschildert werden, wenn nicht nach den vorliegenden neuen medizinischen Erkenntnissen das Karpaltunnelsyndrom auch bei intensivem Smartphone-Gebrauch auftreten würde. In Hongkong wurde im Jahr 2017 bei Studenten gefunden, dass diejenigen mit mehr als 5 Stunden Smartphone-Gebrauch täglich signifikant mehr Schmerzen im Bereich der dominanten Hand hatten als Studenten mit weniger als 5 Stunden Smartphone-Nutzung [15]. Auch waren die beiden Tests (Hoffmann-Tinel-Zeichen und Phalen-Zeichen) bei signifikant mehr Studenten der Gruppe der Vielnutzer positiv im Vergleich zur Gruppe der Wenignutzer. Die Untersuchung der Enge des Karpaltunnels mittels Ultraschall zeigte bei den Vielnutzern einen geschwollenen Nerv und damit eine gegenüber den Wenignutzern vermehrte Enge.

Was kommt nun zuerst, das KTS, das sich dann durch das Smartphone verschlimmert, oder ist es das Smartphone, dass das KTS überhaupt erst entstehen lässt? Bereits im Jahr 2016 war - ebenfalls mittels Ultraschall - von der gerade zitierten Arbeitsgruppe gefunden worden, dass ganz bestimmte Handbewegungen, wie sie beim Smartphone-Gebrauch häufig sind, die Enge im Tunnel verstärken und somit das Syndrom hervorrufen können [16]. Diese Handbewegungen kommen vor allem beim einhändigen Gebrauch des Smartphones vor. Bei gleichzeitigem Tippen mit dem Daumen wechseln Extension und Flexion des Daumens rasch ab, und von genau dieser Bewegungen ist bekannt, dass sie zu wiederholten Verengungen des Karpaltunnels und damit zu erhöhtem Druck im Karpaltunnel führen. Die Autoren der Studien rieten allein daher zur Zurückhaltung beim Smartphone-Gebrauch. Diese Studie scheint zu zeigen, dass generell eine Prädisposition besteht (im Sinne einer Enge, die 
ohne Smartphone subklinisch bleibt, wie man es ausdrücken könnte), die durch Bewegungen, die der Smartphone-Gebrauch mit sich bringt, zum klinischen Syndrom führt.

Bereits aus dem Jahr 2015 stammt zudem eine türkische Studie [6], die ebenfalls Hinweise auf eine Verursachung des KTS durch das Smartphone geliefert hat. Insgesamt 202 Studenten wurden nach dem Ausmaß ihrer Smartphone-Nutzung in 3 Gruppen eingeteilt, die Nichtnutzer, die Wenignutzer und die Vielnutzer. Bei der letztgenannten Gruppe wurde eine signifikante Erhöhung der Schmerzen bei Bewegung sowie eine beim Vergleich der dominanten Hand gegen die nicht dominante Hand besonders starke Verdickung der Sehne des Musculus flexor pollicis longus gefunden. Ebenso war - wieder im Seitenvergleich - der Nervus medianus bei den starken Smartphone-Nutzern am stärksten verdickt. Die Autoren heben besonders hervor, dass die Schere der Symptomatik mit dem Ausmaß der Smartphone-Sucht bei den Smartphone-Nutzern zusammenhing. Die Autoren diskutieren ihre Ergebnisse dahingehend, dass eine starke Smartphone-Nutzung das Risiko eines KTS erhöhen könnte. In Ihren Worten: „Zusammenfassend haben wir in dieser Studie einen Zusammenhang zwischen Smartphone-Sucht und einem vergrößerten Nervus medianus gezeigt [...]. Unsere Daten zeigen, dass die übermäßige Nutzung des Smartphones bzw. die Smartphone-Sucht bei prädisponierten Individuen zur Entwicklung eines KTS führen kann “ [6]¹.

Man ist geneigt, dem Smartphone bei der Verursachung eines KTS keinen besonderen Stellenwert einzuräumen, da es so klein und leicht ist. Es ist jedoch keineswegs der Fall, dass nur „schwere Arbeit" ein KTS nach sich zieht. Ganz im Gegenteil: Eine erst kürzlich publizierte prospektive kontrollierte Studie an 1201 Arbeitern, von denen 106 ein KTS aufwiesen, ergab ein schlechteres Ansprechen der Behandlung gerade bei Arbeitern, deren Tätigkeiten wenig Kraftanstrengung im Bereich der Hand erforderten [2]. Bei Grundschulkindern wurden übrigens keine Unterschiede in der Häufigkeit eines KTS bei der Verwendung digitaler Geräte zum Lesen im Vergleich zu Büchern gefunden [11]. Dies war jedoch auch nicht zu erwarten, kommt doch das KTS ohnehin bei Kindern praktisch nicht vor.

Zusammenfassend lässt sich feststellen, dass das KTS - neben vielen anderen Erkrankungen, wie Kurzsichtigkeit, Schlafstörungen oder Depressionen - in die Liste der unerwünschten Auswirkungen von übermäßiger Smartphone-Nutzung eingereiht werden kann.

1 „In conclusion, in this study we have demonstrated that enlarged median nerves are linked to addiction level [...]. The data suggest that smartphone overuse/addiction may lead prone individuals to develop CTS.“
Literatur

[1] Bower JA, Stanisz G], Keir PJ. An MRI evaluation of carpal tunnel dimensions in healthy wrists: Implications for carpal tunnel syndrome. Clin Biomech 2006; 21: 816-825

[2] Cardona A, Thiese MS, Kapellusch J, et al. Role of Biomechanical Factors in Resolution of Carpal Tunnel Syndrome Among a Population of Workers. J Occup Environ Med 2019; 6: 340-346

[3] Eitivipart AC, Viriyarojanakul S, Redhead L. Musculoskeletal disorder and pain associated with smartphone use: A systematic review of biomechanical evidence. Hong Kong Physiother J 2018; 38: 77-90

[4] IJmker S, Huysmans MA, Blatter BM, et al. Should office workers spend fewer hours at their computer? A systematic review of the literature. Occup Environ Med 2007; 64: 211-222

[5] IJmker S, Huysmans MA, van der Beek A], et al. Software-recorded and self-reported duration of computer use in relation to the onset of severe arm-wrist-hand pain and neck-shoulder pain. Occup Environ Med 2011; 68: 502-509

[6] Inal EE. Effects of smartphone overuse on hand function, pinch strength, and the median nerve. Muscle Nerve 2015; 52: 183-118

[7] $\mathrm{Kim} \mathrm{HJ}$, Kim JS. The relationship between smartphone use and subjective musculoskeletal symptoms and university students. J Phys Ther Sci 2015; $27: 575$

[8] Lakshminarayanan K, Shah R, Li Z-M. Sex-related differences in carpal arch morphology. PLoS ONE 2019; 14(5): e0217425. https://doi. org/10.1371/journal.pone.0217425

[9] Lee YS, Yang HS, Jeong C], et al. Changes in the thickness of median nerves due to excessive use of smartphones. J Phys Ther Sci 2012; 24 (12): 1259-1262

[10] Mediouni Z, Bodin J, Dale AM, et al. Carpal tunnel syndrome and computer exposure at work in two large complementary cohorts. BM] Open 2015; 5: e008156. doi:10.1136/bmjopen-2015-008156

[11] Seomun G, Pyun SB, Lee JA, et al. A comparison of carpal tunnel syndrome between digital and paper textbook users in elementary schools. Work 2016; 53: 909-915

[12] Shim JM. The effect of carpal tunnel changes on smartphone users. J Phys Ther Sci 2012; 24 (12): 1251-1253

[13] Toh SH, Coenen P, Howie EK, et al. The associations of mobile touch screen device use with musculoskeletal symptoms and exposures: A systematic review. PLoS ONE 2017; 12(8): e0181220. doi. org/10.1371/journal. pone. 0181220

[14] Woo EHC, White P, Lai CWK. Impact of information and communication technology on child health. J Paediatr Child Health 2016; 52: 590-594

[15] Woo EHC, White P, Lai CWK. Effects of electronic device overuse by university students in relation to clinical status and anatomical variations of the median nerve and transverse carpal ligament. Muscle Nerve 2017; 56: 873-880

[16] Woo EHC, White P, Ng HK, et al. Development of Kinematic Graphs of Median Nerve during Active Finger Motion: Implications of Smartphone Use. PLoS ONE 2016; 11(7): e0158455. doi:10.1371/journal. pone. 0158455 